\title{
Induced epidural haematoma
}

\author{
Sherief Ahmed El Anany, Abd Elghany Elshamy
}

\author{
Mansoura University, EGYPT
}

\section{ABSTRACT}

Background: A novel case of epidural haematoma caused by general surgery

Case presentation: Fifty-four years male patient was admitted at Mansoura Emergency Hospital with Glasgow coma scale 4 on ventilator. rapid evacuation of EDH was done and the GCS became 9

Conclusion: rapid accumulation of epidural haematoma is fatal and may lead to death.

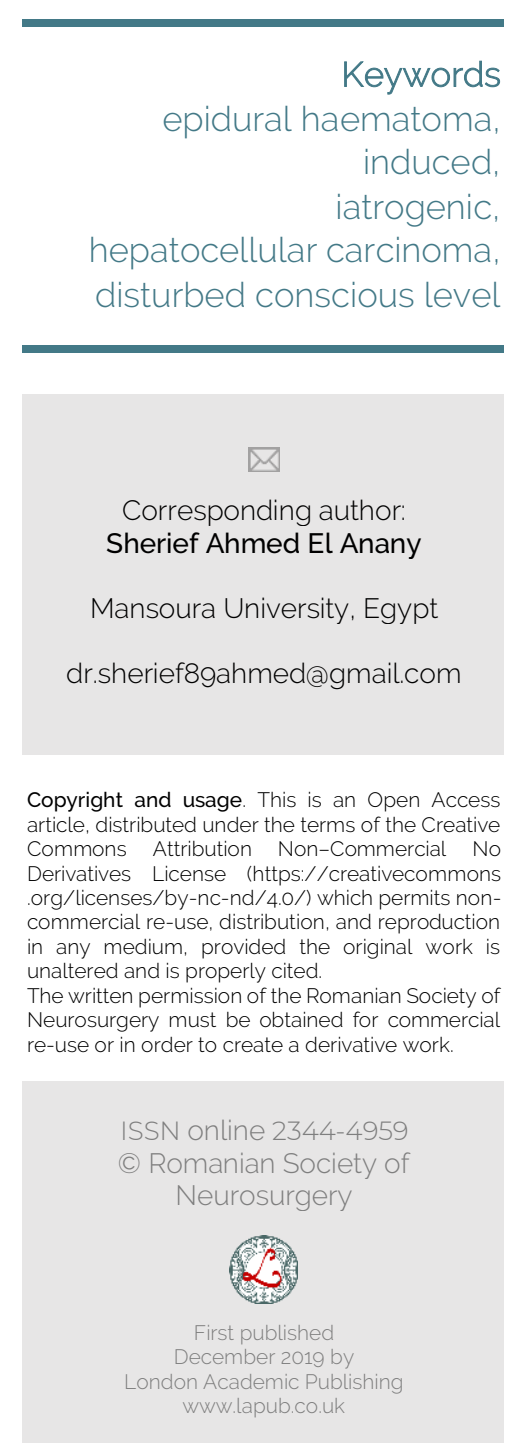

\section{BACKGROUND}

An epidural hematoma (EDH) is an extra-axial collection of blood within the potential space between the outer layer of the dura mater and the inner table of the skull. It is confined by the lateral sutures (especially the coronal sutures) where the dura inserts. It is a life-threatening condition, which may require immediate intervention and can be associated with significant morbidity and mortality if left untreated. Rapid diagnosis and evacuation are important for a good outcome. It occurs in approximately $10 \%$ of traumatic brain injuries (TBI) requiring hospitalization. Both by traumatic and non-traumatic mechanisms can cause an epidural hematoma. The majority of cases related to a traumatic mechanism are a result of head injury due to motor vehicle collisions, physical assaults, or accidental falls (1). Non-traumatic mechanisms include the following:

- Infection/Abscess

- Coagulopathy

- Haemorrhagic Tumours

- Vascular Malformation

Most epidural hematomas result from arterial bleeding from a branch of the middle meningeal artery. The anterior meningeal artery or dural arteriovenous (AV) fistula at the vertex may be involved. Up to $10 \%$ of EDHs are due to venous bleeding following the laceration of a dural venous sinus. (1)

A novel and rare case in our practice had been developed showing induced epidural haematoma from left temporal soft mass, eroding 
bone metastasis in skull, extending to subgalea and compressing the dura. The aim of this case can be used as a guidance for every practitioner to be cautious when dealing with any head swelling.

\section{CASE PRESENTATION}

Fifty-four years male patient admitted at Mansoura Emergency Hospital with GCS 4 on ventilator. Urgent CT brain was done and showed large epidural hematoma with extra bony lesion (figure.1). Before that incident with three hours, the patient was fully conscious and he visited outpatient clinic where a general surgeon tried to aspirate left temporal soft lesion, causing skin swelling, doughted that it was subgaleal haematoma.

\section{OpERATION}

A left pteriyonal approach was done then a craniotomy with four burr holes. The soft lesion was excised completely with evacuation of EDH (figure.2)
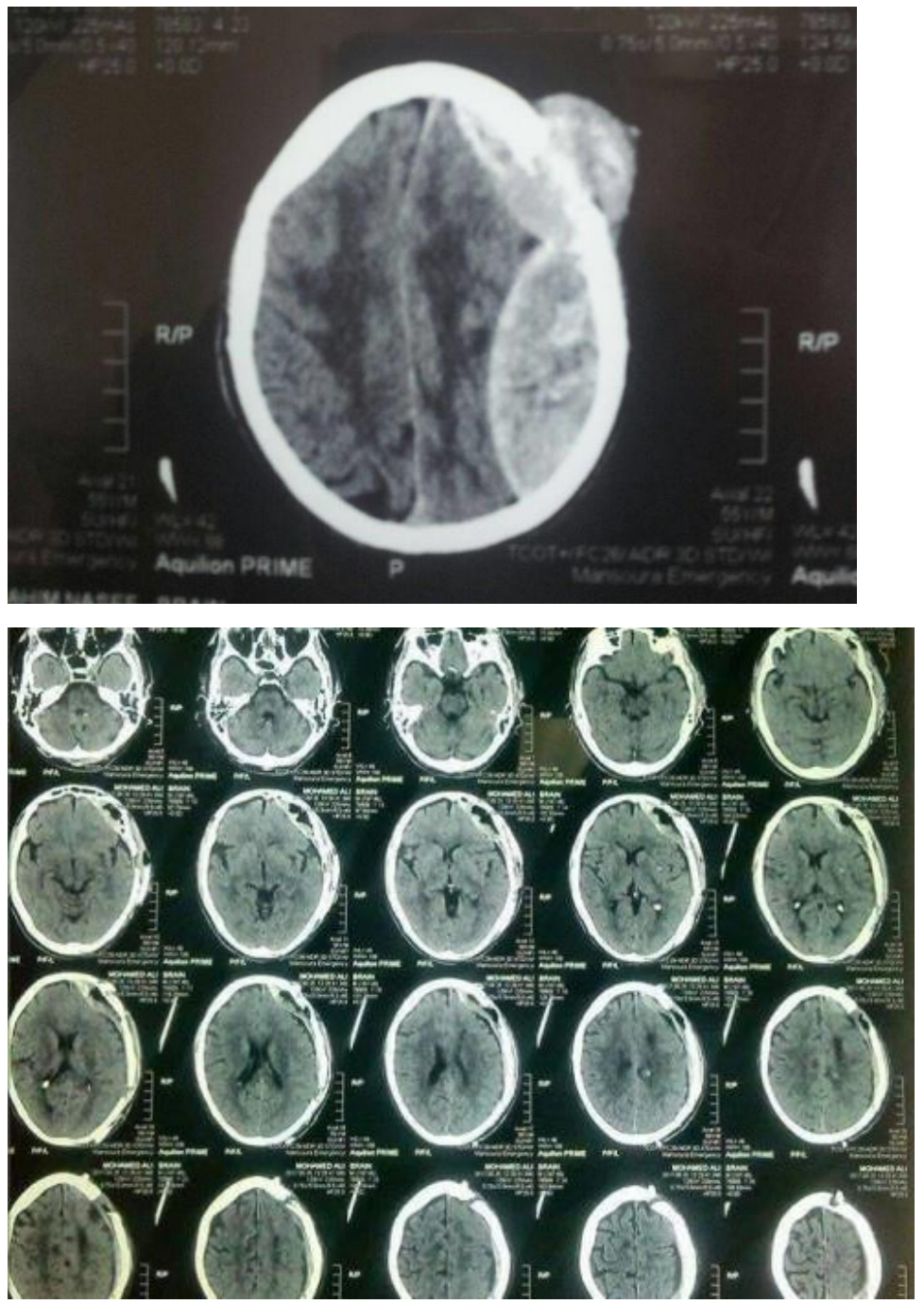

Figure 2: Post-operative CT brain explaining large EDH with bony erosion and extra dural lesion totally removed and the bone was repositionned with closure of skin. the lesion was sent to histopathological analysis and showed hepatocellular carcinoma. The patient remained in ICU with GCS 9 postoperative one week then died.

\section{CONCLUSION}

Epidural hematoma is a relatively common presentation to the emergency department, and if not diagnosed is associated with a high mortality. The condition is best managed by a multidisciplinary team that includes the emergency room physician, the trauma team, radiologist, neurologist, neurosurgeon, intensivist and the ICU nurses. The condition has been associated with mortality rates in excess of $15 \%$. Every practitioner not related to neurosurgery should be careful when dealing with any case with head swelling and refer the case to a specialist avoiding iatrogenic death of patient.

Figure 1: Pre-operative CT brain explaining large EDH with bony erosion and extra dural lesion 
LIST OF ABBREVIATION

GCS : Glasgow coma scale

ICU : intensive care unit

EDH : epidural haematoma

TBI : traumatic brain injuries

$\mathrm{CT}$ : computerized tomography

$\mathrm{AV}$ : arterio venous

\section{DECLARATIONS}

\section{Acknowledgement}

No other person contributed to this article

\section{Funding}

Nothing to disclose

\section{Authors' contribution}

The corresponding author and co-authors performed all of the procedures, clinical assessment, follow-up of patients, collection of results and are responsible for the study conception and design.

\section{Consent for publication}

This study reported no personal data for any patients; informed consents were obtained for all patients included in this study for the publishing of results.

None of the patient involved in this study had declined the publication of study results.

\section{Competing interests}

no conflict of interests.

Availability of data materials Raw data are available

\section{REFERENCES}

Khairat A, Waseem M. Epidural Hematoma. [Updated 2018 Nov 15]. In: StatPearls [Internet]. Treasure Island (FL): StatPearls Publishing; 2019 Jan. Available from: https://www.ncbi.nlm.nih.gov/books /NBK518982/. 